\title{
Acute MI Versus Takotsubo Cardiomyopathy
}

\author{
S. V. V. Mani Krishna ${ }^{1}$ \\ ${ }^{1}$ Department of Cardiology, NIMS, Punjagutta, Hyderabad, \\ India62130135
}

Address for correspondence S. V. V. Mani Krishna, DM, Department of Cardiology, NIMS, Punjagutta, Hyderabad - 500082, India (e-mail: manikrishnas009@gmail.com).

Ind J Car Dis Wom 2021;6:130-135.

\begin{abstract}
Keywords

- Takotsubo cardiomyopathy

- Discussant: Dr. S.V.V. Mani Krishna

- The likely differential diagnoses are as follows:

A 37-year-old female, who was diagnosed with rheumatoid arthritis (RA) 20 years earlier and on regular treatment, with a recent history of pulmonary thromboembolism on inj. clexane, presented with anginal type of chest pain of 4 hours duration ECG, showing ST elevation, and was finally diagnosed to have Takotsubo cardiomyopathy.
\end{abstract}

Presenter: A 37-year-old female, who was a known case of RA since 20 years was on regular treatment with disease-modifying antirheumatic drugs (DMARDs) methotrexate and hydroxychloroquine.

She underwent total knee replacement in 2010 for bilateral knees and total hip replacement in 2012. She also had a history of irregular menstrual cycles, with excessive bleeding from the past one year. While evaluation, she was found to have fibroid uterus, which was planned for surgery. Just before surgery, she developed sudden onset of breathlessness associated with chest pain. She was admitted and evaluated at our center and found to have bilateral lower limb deep vein thrombosis (DVT) with submassive pulmonary thromboembolism. As she has to undergo hysterectomy surgery, so she underwent inferior vena cava (IVC) filter implantation through right internal jugular vein (IJV) access and was discharged with inj. clexane $40 \mathrm{mg}$ subcutaneously (SC) twice daily at home.

After 4 days of discharge from hospital, she was again admitted to our hospital with a sudden onset of chest pain of 4-hour duration, retrosternal in location and deep constricting type, radiating to left upper limb, associated with breathlessness and sweating not related to posture and respiration and food intake lasting for 5 minutes Patient did not

DOI https://doi.org/ $10.1055 / \mathrm{s}-0041-1732511$ give any history of orthopnea or paroxysmal nocturnal dyspnea (PND) episodes.

Neither was there any history of palpitations and giddiness episodes nor of syncope or fall.

There was no history of pedal edema, fever, rash, cough, easy fatiguability, weight loss, abdominal distension or pain, generalized anasarca, constipation, diarrhea, melena, tingling numbness of limbs, reduced urine output, burning micturition, or bleeding manifestations.

There was no history of similar episodes in the past. Patient is housewife by profession. Patient consumes a mixed diet. There was no history of alcohol intake, smoking, or tobacco chewing. There was no history of use of any other medication other than drugs prescribed for rheumatoid arthritis (RA), and inj. clexane prescribed for pulmonary thromboembolism. No family history of any cardiac disease or other medical illness. First childbirth involved intrauterine death at term pregnancy. Second childbirth was uneventful, and child is healthy.

Discussant: Summary of the present illness is a 37-year-old female, with a diagnosed case of RA since 20 years and on regular treatment, along with a recent history of pulmonary thromboembolism on inj. clexane, who presented with anginal type of chest pain of 4 hours duration.

(C) 2021. Women in Cardiology and Related Sciences.

This is an open access article published by Thieme under the terms of the Creative Commons Attribution-NonDerivative-NonCommercial-License, permitting copying and reproduction so long as the original work is given appropriate credit. Contents may not be used for commercial purposes, or adapted, remixed, transformed or built upon. (https://creativecommons.org/licenses/by-nc-nd/4.0/).

Thieme Medical and Scientific Publishers Pvt. Ltd. A-12, 2nd Floor, Sector 2, Noida-201301 UP, India 


\section{Acute Myocardial Infarction}

Chest pain here is characteristic of angina. Myocardial ischemia usually occurs in the setting of coronary atherosclerosis, but it may also reflect dynamic components of coronary vascular resistance. Coronary spasm can occur in normal coronary arteries, in patients with coronary artery disease (CAD), near atherosclerotic plaque, and in smaller coronary arteries. ${ }^{1}$ Other, less common causes of impaired coronary blood flow include syndromes that compromise the orifices or lumina of the coronary arteries, such as coronary arteritis, proximal aortitis, spontaneous coronary artery dissection, proximal aortic dissection, coronary emboli from infectious or noninfectious endocarditis or thrombus in the left atrium or left ventricle, myocardial bridge, or a congenital abnormality of the coronary arteries.

\section{Acute Pulmonary Thromboembolism}

Massive pulmonary emboli tend to cause severe and persistent substernal pain, which is attributed to distention of the pulmonary artery. ${ }^{2}$ Smaller emboli that lead to pulmonary infarction can cause lateral pleuritic chest pain. Hemodynamically significant pulmonary emboli may cause hypotension, Syncope and right-sided heart failure. In our patient, there is no syncope and right-sided heart failure.

\section{Acute Myopericarditis}

Pericarditis patients typically experience pleuritic pain with breathing, coughing, and changes in position. Swallowing may induce the pain because of the proximity of the esophagus to the posterior portion of the heart. Because the central diaphragm receives its sensory supply from the phrenic nerve and the phrenic nerve arises from the third to fifth cervical segments of the spinal cord, pain from pericardium is frequently felt in the shoulders and neck. Our patient does not have chest pain characteristic of pericarditis. However, pericarditis occasionally causes a steady, crushing substernal pain resembling that of acute myocardial infarction (AMI). ${ }^{3}$

\section{Acute Aortic Dissection}

Acute aortic dissection usually causes a sudden onset of excruciating ripping pain. Ascending aortic dissection tends to manifest as pain in the midline of the anterior aspect of the chest, and posterior descending aortic dissection tends to cause pain in the back of the chest. ${ }^{4}$ Our patient does not have any backpain and ripping quality.

\section{Severe PAH Secondary to Rheumatoid Arthritis}

As patient is a known case of RA since 20 years, chances of her having pulmonary arterial hypertension (PAH) is high. PAH can result in chest pain, similar to that of angina pectoris, presumably because of right heart hypertrophy and ischemia. $^{5}$

\section{Takotsubo Cardiomyopathy}

Patient had emotional triggers because of multiple surgeries for RA, so chances of developing Takotsubo cardiomyopathy is high. Chest pain is the predominant symptom (around 76\%) in Takotsubo cardiomyopathy. ${ }^{6}$

\section{Spontaneous Pneumothorax}

Sudden onset of unilateral pleuritic pain, with dyspnea is known to occur in spontaneous pneumothorax. However, in our patient, chest pain is retrosternal and not unilateral.

\section{Esophageal Spasm}

Esophageal spasm can produce a squeezing chest discomfort, similar to that of angina. Patient is also having associated breathlessness, so likely possibility of esophageal spasm is very less.

Presenter: On examination, patient was alert and oriented. Her body mass index (BMI) was $32.6 \mathrm{~kg} / \mathrm{m}^{2}$. Temperature was $98.4^{\circ} \mathrm{F}$. Pulse rate was 100 beats/min, which was regular; blood pressure was $110 / 70 \mathrm{~mm} \mathrm{Hg}$ in right arm supine position. Respiratory rate was 22 cycles per minute and oxygen saturation was $99 \%$ while breathing ambient air. There was no pallor, icterus, cyanosis, clubbing, edema feet, or lymphadenopathy. Deformities of small joints of hands and feet were present ( - Figs. 1 A, B). On cardiovascular system examination, jugular venous pressure (JVP) had normal mean column height and normal waveforms. Apex beat was localized in left 5th intercostal space along midclavicular line, left ventricular (LV) type and diffuse impulse. First heart sound was soft in intensity. LV S3 and S4 are audible. Second heart sound was normal in intensity and split. No adventitious sounds were appreciated. The lungs were clear. There was no organomegaly as per abdomen examination.

Discussant: To summarize the examination findings, patient had a pulse rate of $100 / \mathrm{min}$, regular with a normal JVP and soft intensity of first heart sound, and LV S3 and S4 pointing toward AMI as the cause of acute chest pain.

Comprising history and examination findings, the probable diagnosis is as follows:

\section{Acute Myocardial Infarction}

Patients with RA are prone to have early atherosclerosis. Systemic inflammatory diseases cause premature atherogenesis. Evidence supporting an association between
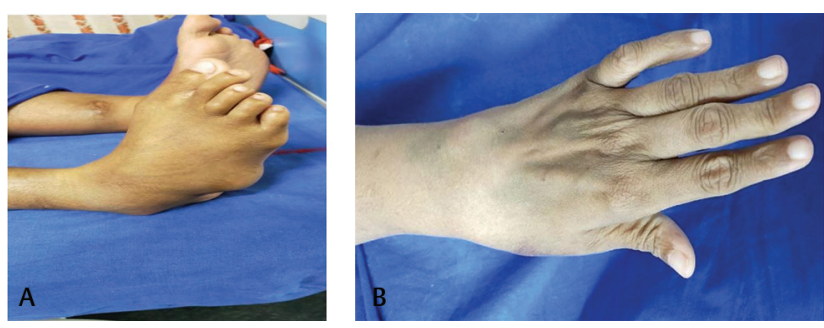

Fig. 1 (A, B) Showing deformities of small joints of feet and hands. 
inflammatory diseases and accelerated atherogenesis is best developed for RA and systemic lupus erythematosus (SLE). ${ }^{7}$ The risk of myocardial infarction in patients with RA is considered similar to that in those with diabetes mellitus, and women with RA are twice as likely as age-matched controls in the general population to suffer myocardial infarction. ${ }^{8}$ Coronary arteritis can also occur in RA.

\section{Acute Pericarditis}

In more than $90 \%$ of cases, the main symptom of acute pericarditis is chest pain, often quite severe. It is usually retrosternal. The trapezius ridge is a classic radiation. Pericardial pain is pleuritic and worsened by lying down. Associated symptoms include dyspnea, cough, and occasionally hiccups.

Clinically, significant pericarditis affects only 1 to $2 \%$ of patients with RA, more commonly male, seropositive patients. ${ }^{9}$ Constrictive pericarditis can develop over a period of months. Hemodynamically significant pericarditis, although reported, is extremely rare in patients being treated with antirheumatic therapy.

Viral Myocarditis: Patient presented with sudden onset shortness of chest pain along with breathlessness. Hence, the possibility of viral myocarditis should be strongly considered. However, no inciting event was reported by the patient.

\section{Takotsubo Cardiomyopathy}

Chest pain was the predominant symptom in $76 \%$, dyspnea in $47 \%$, and syncope in $7.7 \%$. A preceding physical trigger occurred in $36 \%$ and an emotional trigger in $28 \%{ }^{10}$

Investigations:

1. Chest X-ray (-Fig. 2)

2. ECG

a. ECG at the time of admission ( - Fig. 3 )

b. ECG on day 3 of admission ( - Fig. 4 )

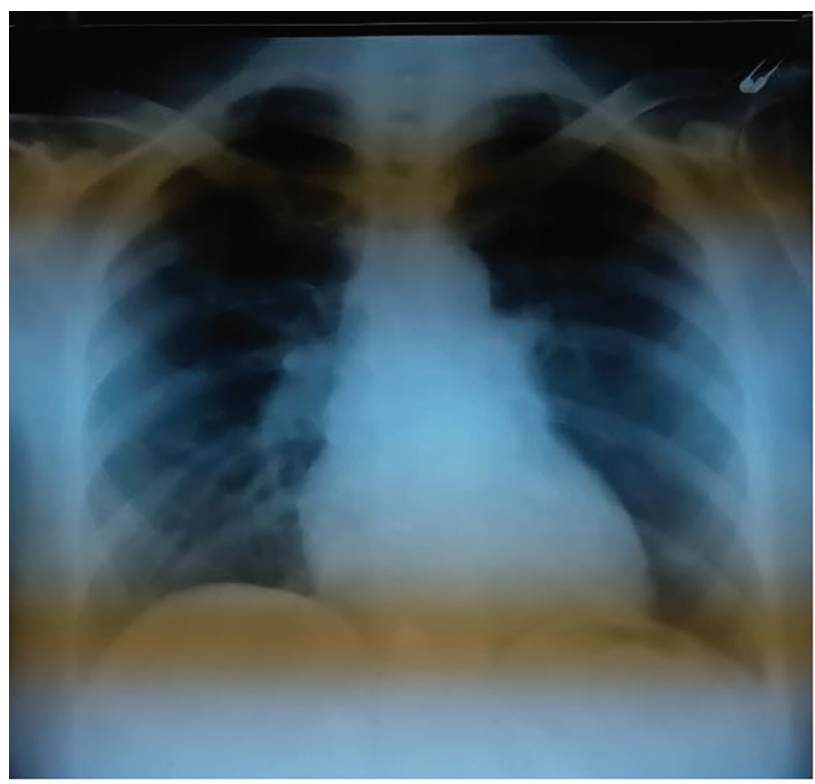

Fig. 2 Chest X-ray PA view.
3. 2D echo ( - Figs. 5 and $\mathbf{6})$

4. Laboratory investigations ( - Table $\mathbf{1}$ )

5. Others.

Radiologist: Chest X-ray showing cardiomegaly with LV apex ( - Fig. 1). Aorta is normal, and pulmonary bay is preserved. Grade 1 pulmonary venous hypertension.

\section{Electrophysiologist Opinion}

ECG on admission was suggestive of anterior wall AMI with ST elevation in all the precordial leads 1 and augmented Vector Left $(\mathrm{aVL})(\boldsymbol{-}$ Fig. $\mathbf{3})$.

Table 1 Laboratory investigations

\begin{tabular}{|c|c|}
\hline Biochemical parameter & Patient range \\
\hline Hemoglobin (gm/dl) & 14 \\
\hline White blood cells (per ul) & 8600 \\
\hline Platelet count (per ul) & 260000 \\
\hline $\begin{array}{l}\text { Sodium/potassium } \\
\text { (mmol/L) }\end{array}$ & $137 / 5.1$ \\
\hline Creatinine/urea (mg/dl) & $0.86 / 50$ \\
\hline Total bilirubin/direct (mg/dl) & $0.6 / 0.2$ \\
\hline SGOT/SGPT (U/L) & $36 / 28$ \\
\hline $\begin{array}{l}\text { Total protein/albumin (gm/ } \\
\text { dl) }\end{array}$ & $8 / 4.2$ \\
\hline PT/INR/APTT (sec) & $11 / 1.02 / 24$ \\
\hline $\begin{array}{l}\text { Total cholesterol/TGC (mg/ } \\
\text { dl) }\end{array}$ & $152 / 78$ \\
\hline HDL/LDL/VLDL (mg/dl) & $42 / 84 / 16$ \\
\hline Hs-CRP (mg/L) & 2 \\
\hline ESR (mm 1 hour) & 28 \\
\hline NT pro BNP (pg/ml) & 12116 \\
\hline ANA profile & Negative \\
\hline CPK/LDH (U/L) & $400 / 243$ \\
\hline Peak troponin T levels & $26 \mathrm{ng} / \mathrm{L}$ \\
\hline Antiphospholipid antibodies & Negative \\
\hline Plasma homocysteine & $18 \mathrm{mcmol} / \mathrm{L}$ \\
\hline
\end{tabular}

Abbreviations: ANA, antinuclear antibody; APTT, activated partial thromboplastin time; CPK, creatine phosphokinase; CRP, C-reactive protein; ESR, erythrocyte sedimentation rate; HDL, high-density lipoprotein; INR, international normalized ratio; LDH, lactate dehydrogenase; LDL, low-density lipoprotein; PT, prothrombin time; TGC, triglycerides; VLDL, very low density lipoprotein.

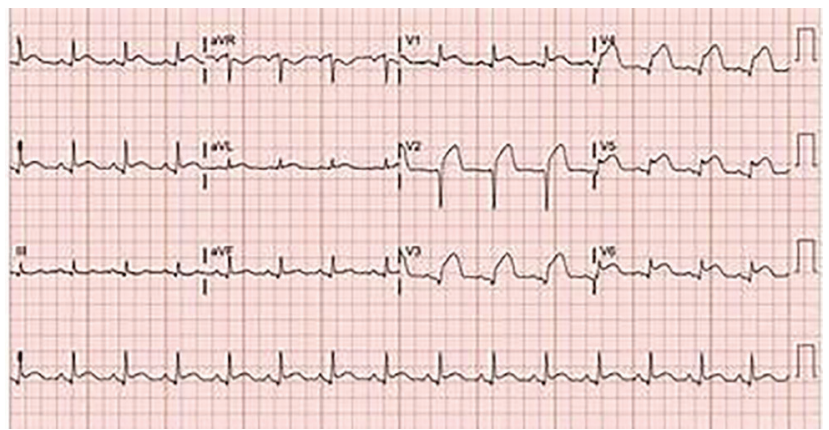

Fig. 3 ECG on hospital admission. 


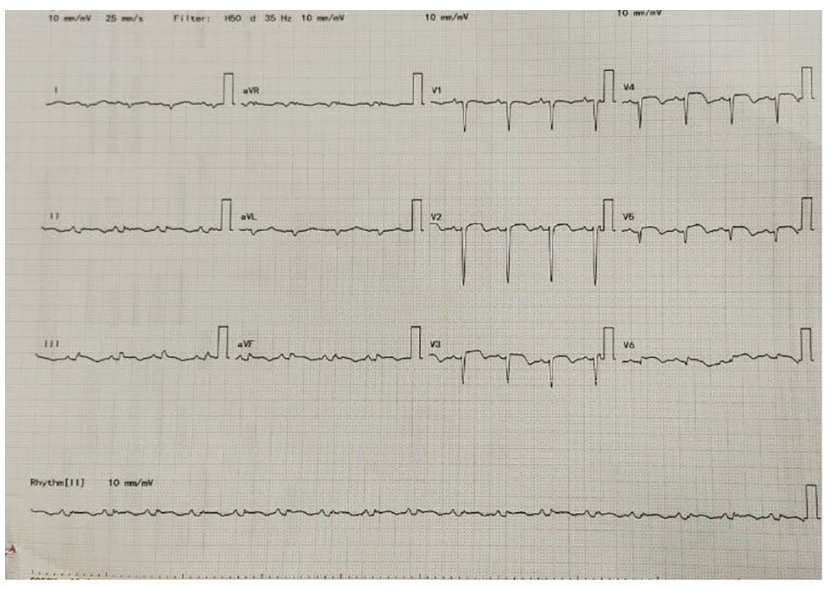

Fig. 4 Follow-up ECG on day 3 of hospital stay after thrombolysis with tenecteplase.

As patient is presented within 4 hours of chest pain, she underwent thrombolysis with tenecteplase. Following thrombolysis, there is resolution of ST elevations ( - Fig. 4).

\section{Discussant}

So, after chest X-ray and ECG, there are three possible differential diagnosis.

\section{Acute Myocardial Infarction}

Evolution of ECG changes and development of Q waves in our patient points toward myocardial infarction as the possible etiology of chest pain. But there is absence of reciprocal ST segment depressions. There is 2 to $3 \mathrm{~mm}$ ST elevation in precordial leads along with $\mathrm{Q}$ waves.

\section{Acute Pericarditis}

Pericarditis will have ST-segment elevation in all leads except augmented Vector Right (aVR) and often V1. Usually, the ST segment is coved upward and resembles transmural ischemia. Both can be differentiated by more extensive lead involvement and lack of evolution to pathologic $Q$ waves in pericarditis, and more prominent reciprocal ST depression in ischemia. ${ }^{11}$ PR segment depression is also common and considered the earliest ECG sign of acute pericarditis. ${ }^{11}$ In our case, ECG evolution to $Q$ waves is seen and there is no PR segment depression. So, the possibility of acute pericarditis is less likely.

\section{Takotsubo Cardiomyopathy}

ST elevation shown on the ECG in almost half of the patients with Takotsubo cardiomyopathy. New and reversible ECG abnormalities (ST-segment elevation, ST depression, LBBB, b T-wave inversion, and/or QTc prolongation) are seen during the acute phase (first 3 months). ${ }^{12}$

Presenter: 2D echo was done and suggestive of regional wall motion abnormality (RWMA) in anterior wall and apex, mild LV dysfunction ejection fraction (EF) $42 \%$, grade I diastolic dysfunction, no mitral regurgitation (MR)/aortic regurgitation (AR)/tricuspid regurgitation (TR)/PAH, and no pulmonary embolism (PE)/vegetation/clot ( - Fig. 5).

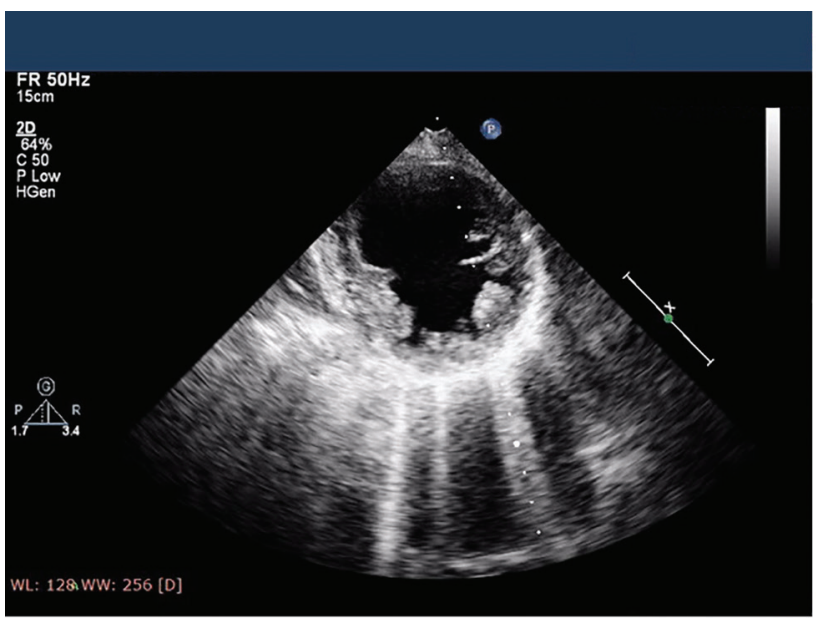

Fig. 5 Echo image showing mild left ventricle (LV) dysfunction at the time of admission.

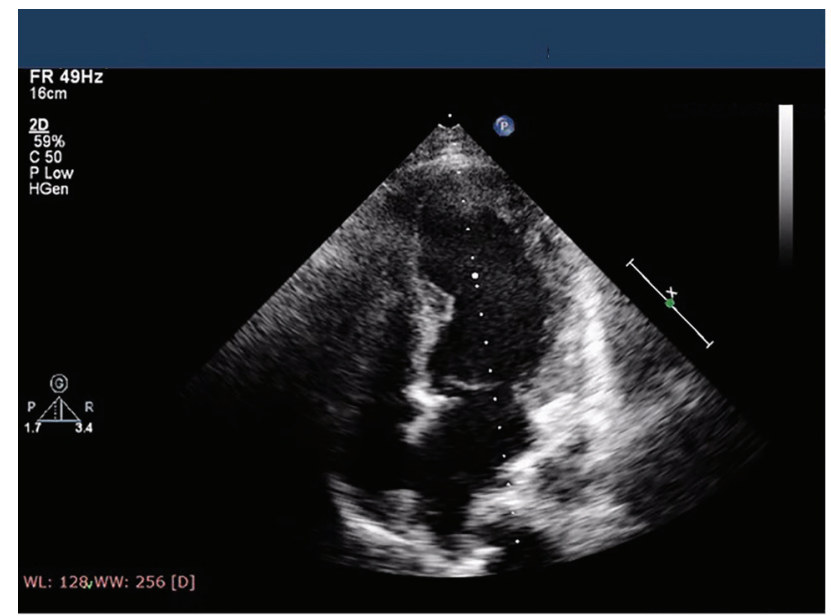

Fig. 6 Showing severe left ventricle (LV) dysfunction on day 2 of admission with apical ballooning.

Presenter: Following thrombolysis with tenecteplase, patient developed profuse vaginal bleeding not controlled by medical therapy. She also developed pulmonary edema requiring continuous positive airway pressure (CPAP) support. 2D echo at that time has shown severe LV dysfunction with apical ballooning (-Fig. 6). Gynecology consultation taken for profuse vaginal bleeding were advised uterine artery embolization. Patient underwent successful uterine artery embolization with control of vaginal bleeding.

The possible differential diagnoses based on 2D echo findings are as follows:

\section{Takotsubo Cardiomyopathy}

In our case, there is circumferential dysfunction of ventricular segments, and abnormality is not confined to single vascular territory. These features are favoring Takotsubo cardiomyopathy. The RWMAs usually extend beyond a single epicardial vascular distribution and cause circumferential dysfunction of the ventricular segment in case of Takotsubo cardiomyopathy..$^{13} \mathrm{LV}$ contractile abnormalities in Takotsubo 
cardiomyopathy are prominent, and although they involve the LV apex (resulting in the synonym of "apical ballooning syndrome") in more than $80 \%$ of patients, RWMAs may be limited to the midventricular wall or other LV walls in a minority of patients. ${ }^{13}$

\section{Acute Myocardial Infarction}

As $2 \mathrm{D}$ echo is showing wall motion abnormalities in apical areas, even though not confined to single vascular territory, still the possibility of AMI cannot be denied in our case.

Presenter: Laboratory investigations

Except for elevation of cardiac enzymes and NT pro BNP, the rest of the reports were normal.

\section{Takotsubo Cardiomyopathy}

In our case, there is disparity between peak troponin levels and amount of dysfunctional myocardium. Also, there exists an inverse relation between NT pro BNP levels and peak troponin T levels, with NT pro BNP levels being significantly elevated compared with troponin T. These features favor Takotsubo cardiomyopathy rather than anterior wall AMI. A positive but relatively small elevation in cardiac troponin can be measured with a conventional assay in Takotsubo cardiomyopathy. ${ }^{14}$

\section{Acute Myocardial Infarction}

The rise in BNP and NT pro BNP after ST-elevation myocardial infarction (STEMI) correlates with infarct size and RWMAs. But, in our patient, there is mild elevation of cardiac troponin $\mathrm{T}$ and its levels normalized within 1 week. Because of continuous release from a degenerating contractile apparatus in necrotic myocytes, elevations in cTnI may persist for 7 to 10 days after MI; elevations in cTnT may persist for up to 10 to 14 days. ${ }^{15}$ These laboratory findings are against the diagnosis of MI.

As levels of inflammatory markers like erythrocyte sedimentation rate (ESR) and C-reactive protein (CRP) are less in this patient, so the possibility of coronary arteritis as the cause of acute MI is less likely. Antiphospholipid antibodies are also negative in our patient, so the possibility of antiphospholipid syndrome causing coronary thrombosis is ruled out.

\section{Coronary Angiogram}

Coronary angiogram (CAG) was done and showed normal epicardial coronaries ( - Figs. 7 and $\mathbf{8}$ ).

\section{Discussant}

\section{Takotsubo Cardiomyopathy}

Normal epicardial coronary arteries in our case establishes the diagnosis of Takotsubo cardiomyopathy. Diagnosis of Takotsubo cardiomyopathy is certain if there is an absence of culprit atherosclerotic coronary artery disease, including acute plaque rupture, thrombus formation, and coronary dissection or other pathologic conditions, to explain the pattern of temporary LV dysfunction observed (e.g., hypertrophic cardiomyopathy, viral myocarditis). ${ }^{16}$ RWMAs of LV or right ventricle (RV) myocardium occur and are frequently, but not always, preceded by a stressful trigger (emotional or physical).

Emotional triggers are present in our patient because of multiple surgeries for RA and need for another surgery (hysterectomy) in few days.

\section{Acute Anterior Wall MI}

Diagnosis of anterior wall AMI is ruled out in our patient because of normal epicardial coronary arteries. RA is known for premature atherosclerosis, but in our patient, there is no evidence of plaque in coronary angiogram, so plaque rupture, erosion and thrombosis are ruled out. There is no luminal irregularities in coronary arteries to suspect coronary arteritis.

\section{Clinical Impression}

Takotsubo cardiomyopathy is the diagnosis in our patient because of minimal elevation of cardiac troponins and more elevation in NT pro BNP, associated with normal coronaries ( - Figs. 7 and $\mathbf{8}$ ) in the presence of emotional trigger. Clinical follow-up after 3 months has shown normal LV function in echocardiography, suggesting reversible dysfunction of LV.

\section{Clinical Diagnosis}

\section{Takotsubo Cardiomyopathy}

Discussion of Management

Takotsubo cardiomyopathy is a self-limited disorder, usually with rapid resolution of the symptoms and LV dysfunction. The patient was treated with $\beta$-blockers and

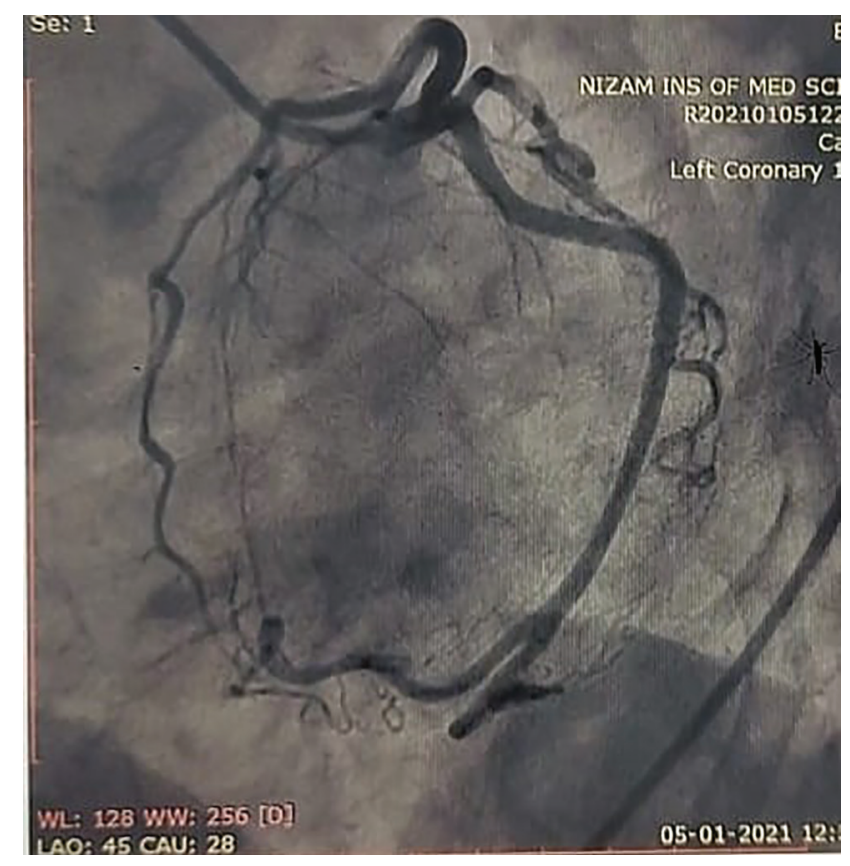

Fig. 7 Coronary angiogram (CAG) in left anterior oblique (LAO) caudal view showing normal proximal left anterior descending (LAD) artery and proximal left circumflex artery (LCX) which are normal. 


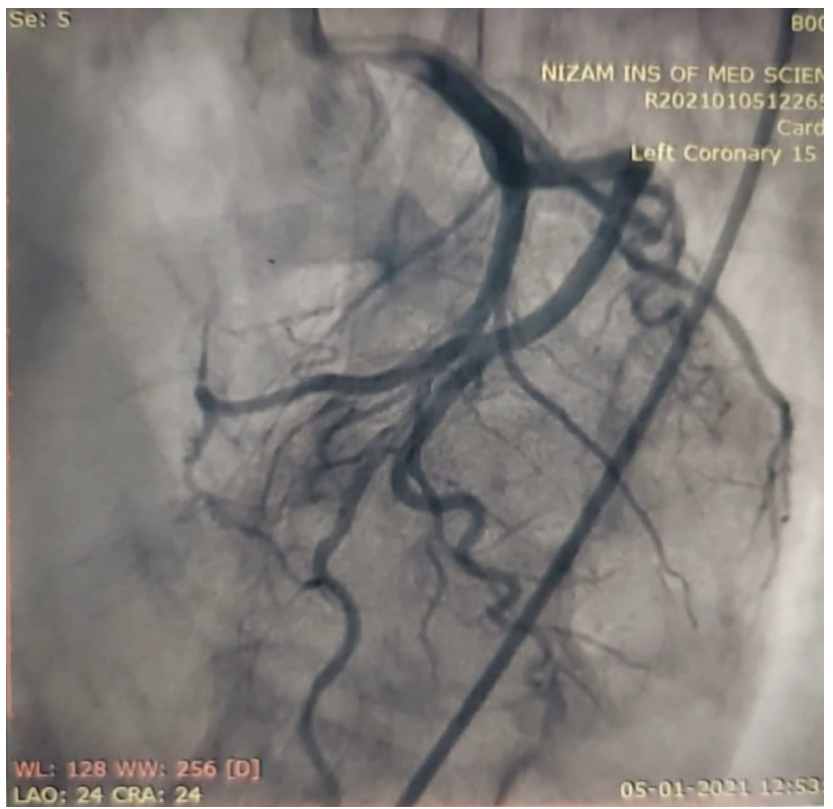

Fig. 8 Coronary angiogram (CAG) in LAO cranial view showing normal mid and distal left anterior descending (LAD) artery.

angiotensin-converting enzyme (ACE) inhibitors. Follow-up after 3 months has shown improvement in symptoms and LV function. Follow-up 2D echo has shown EF of 52\% at 3 months with improvement in functional New York heart Association (NYHA) class from II to III. The European task force position statement suggests classification of patients with Takotsubo cardiomyopathy into lower-risk and higher-risk categories, with the latter based on an LV EF of less than $45 \%$, hypotension and an outflow tract gradient of greater than $40 \mathrm{~mm}$ $\mathrm{Hg}$, and/or the presence of an arrhythmia. ${ }^{17}$ Consideration of an ACE inhibitor and/or a $\beta$-blocker is recommended in the higher-risk groups. ${ }^{17}$ In our patient, $\mathrm{EF}$ is $42 \%$ at the time of admission, so she comes under high-risk category and was treated with ACE inhibitors and $\beta$-blockers. Leading hypothesis suggests that a catecholamine surge results in regional microvascular dysfunction in susceptible patients, accompanied by cellular calcium overload. ${ }^{18}$

\section{Final Diagnosis}

Takotsubo cardiomyopathy.

\section{Conflict of Interest}

None declared.

\section{References}

1 Diamond GA, Forrester JS. Analysis of probability as an aid in the clinical diagnosis of coronary-artery disease. $\mathrm{N}$ Engl J Med 1979;300(24):1350-1358
2 Fedullo P, Kerr KM, Kim NH, Auger WR. Chronic thromboembolic pulmonary hypertension. Am J Respir Crit Care Med 2011;183(12):1605-1613

3 Dudzinski DM, Mak GS, Hung JW. Pericardial diseases. Curr Probl Cardiol 2012;37(3):75-118

4 Hagan PG, Nienaber CA, Isselbacher EM, et al. The International Registry of Acute Aortic Dissection (IRAD): new insights into an old disease. JAMA 2000;283(7):897-903

5 Cottin V. Treatment of pulmonary hypertension in interstitial lung disease: do not throw out the baby with the bath water. Eur Respir J 2013;41(4):781-783

6 Templin C, Ghadri JR, Diekmann J, et al. Clinical features and outcomes of Takotsubo (stress) cardiomyopathy. N Engl J Med 2015;373(10):929-938

7 Solomon DH, Reed GW, Kremer JM, et al. Disease activity in rheumatoid arthritis and the risk of cardiovascular events. Arthritis Rheumatol 2015;67(6):1449-1455

8 Symmons DP. Do we need a disease-specific cardiovascular risk calculator for patients with rheumatoid arthritis? Arthritis Rheumatol 2015;67(8):1990-1994

9 del Rincón I, Polak JF, O'Leary DH, et al. Systemic inflammation and cardiovascular risk factors predict rapid progression of atherosclerosis in rheumatoid arthritis. Ann Rheum Dis 2015;74(6):1118-1123

10 Syed FF, Asirvatham SJ, Francis J. Arrhythmia occurrence with takotsubo cardiomyopathy: a literature review. Europace 2011;13(6):780-788

11 Imazio M, Gaita F. Diagnosis and treatment of pericarditis. Heart 2015;101(14):1159-1168

12 Lyon AR, Bossone E, Schneider B, et al. Current state of knowledge on Takotsubo syndrome: A Position Statement from the Taskforce on Takotsubo Syndrome of the Heart Failure Association of the European Society of Cardiology. Eur J Heart Fail 2016;18(1):8-27

13 Parodi G, Bellandi B, Del Pace S, et al. Tuscany Registry of Tako-Tsubo Cardiomyopathy. Natural history of tako-tsubo cardiomyopathy. Chest 2011;139(4):887-892

14 Bybee KA, Prasad A, Barsness GW, et al. Clinical characteristics and thrombolysis in myocardial infarction frame counts in women with transient left ventricular apical ballooning syndrome. Am J Cardiol 2004;94(3):343-346

15 Weaver JC, Ramsay DD, Rees D, Binnekamp MF, Prasan AM, McCrohon JA. Dynamic changes in ST segment resolution after myocardial infarction and the association with microvascular injury on cardiac magnetic resonance imaging. Heart Lung Circ 2011;20(2):111-118

16 Sharkey SW, Lesser JR, Zenovich AG, et al. Acute and reversible cardiomyopathy provoked by stress in women from the United States. Circulation 2005;111(4):472-479

17 Gianni M, Dentali F, Grandi AM, Sumner G, Hiralal R, Lonn E. Apical ballooning syndrome or takotsubo cardiomyopathy: a systematic review. Eur Heart J 2006;27(13):1523-1529

18 Mori H, Ishikawa S, Kojima S, et al. Increased responsiveness of left ventricular apical myocardium to adrenergic stimuli. Cardiovasc Res 1993;27(2):192-198 